\title{
Associations between serum uric acid and the incidence of hypertension: a Chinese senior dynamic cohort study
}

Fengjiang Wei ${ }^{1 \dagger}$, Ning Sun ${ }^{2 \dagger}$, Chunyou Cai ${ }^{1 \dagger}$, Shuzhi Feng ${ }^{2 \dagger}$, Jianli Tian ${ }^{2}$, Wentao Shi ${ }^{1}$, Weili Xu ${ }^{3}$, Yaogang Wang ${ }^{4^{*}}$, Xilin Yang ${ }^{5^{*}}$ and Wei-Dong $\mathrm{Li}^{\mathrm{i}^{*}}$

\begin{abstract}
Background: The prevalence of hyperuricemia has increased dramatically during the past several decades. Studies indicating uric acid is an independent risk factor for hypertension did not sufficiently control for other known risk factors. We explored this relationship in a comprehensive Chinese senior dynamic cohort.

Methods: To investigate the relationship between serum uric acid (SUA) levels and hypertension, we carried out a 6-year retrospective study (2006-2011) in a dynamic cohort with 3591 subjects free of hypertension. The first occasion of documented hypertension per subject was the index event. A Cox proportional hazards model assessed the relationship between SUA and hypertension. Kaplan-Meier survival analysis compared incidence of hypertension among individuals with each SUA quartile. Receiver operating characteristic curves were generated to obtain the area under the curve as a prediction of hypertension from SUA levels.

Results: The cumulative prevalence of hypertension in our cohort was $20.7 \%$. The prevalence of hyperuricemia was $17.5 \%$. Cox regression analysis showed that, compared with the lowest SUA quartile $(<4.69 \mathrm{mg} / \mathrm{dll})$, the $4.69-5.58$, $5.58-6.52$, and $\geq 6.52 \mathrm{mg} / \mathrm{dl}$ quartiles yielded hazard ratios (95\% confidence intervals) for hypertension of 1.652 (1.265-2.156), 2.195 (1.705-2.825), and 3.058 (2.399-3.899), respectively. Cumulative incidence of hypertension was consistently higher among individuals with hyperuricemia than among those with normal SUA levels. A Kaplan-Meier survival analysis showed that hyperuricemia predicted higher incidences of hypertension in a dose-dependent manner: hypertension onset significantly differed across SUA quartiles. SUA levels were significantly and independently associated with incidence of hypertension in our cohort.
\end{abstract}

Conclusions: Our results, controlling for known risk factors, suggest that SUA level is an independent risk factor for hypertension and could be a useful indicator of hypertension.

Keywords: Hyperuricemia, Hypertension, Dynamic cohort study, Survival analysis

\footnotetext{
*Correspondence: wyg@tmu.edu.cn; yangxilin@tmu.edu.cn;

liweidong98@tijmu.edu.cn

†Fengjiang Wei, Ning Sun, Chunyou Cai and Shuzhi Feng contributed

equally to this paper

${ }^{1}$ Research Center of Basic Medical Sciences, Tianjin Medical University, 22

Qixiangtai Road, Tianjin 300070, People's Republic of China

${ }^{4}$ School of Public Health, Tianjin Medical University, 22 Qixiangtai Road,

Tianjin 300070, People's Republic of China

${ }^{5}$ Department of Epidemiology and Biostatistics, School of Public Health,

Tianjin Medical University, 22 Qixiangtai Road, Tianjin 300070, People's

Republic of China

Full list of author information is available at the end of the article
} 


\section{Background}

Uric acid is the final product of purine metabolism. Serum uric acid (SUA) concentration is precisely regulated, including secretion and reabsorption in the kidneys. Hypertension is highly prevalent, affecting approximately one-third of Americans and is a leading cause of morbidity and mortality [1]. While the etiology of hypertension is unclear in many patients, uric acid has been hypothesized to activate the renin-angiotensin system, which can lead to injury to prerenal blood vessels [2].

A number of epidemiological studies have shown that increased uric acid concentrations are associated with increased risk for developing hypertension [3, 4]. Some observations in cross-sectional analyses and longitudinal studies concluded there was a hyperuricemia-hypertension link [5-9]. Although elevated SUA levels have been predictive of hypertension in longitudinal studies, the relationship between uric acid and blood pressure is confounded by numerous factors, so controversy remains. For example, although elevated uric acid levels are often associated with established traditional cardiovascular risk factors, it is not quite clear whether uric acid is the cause or a consequence of hypertension; studies indicating uric acid as an independent risk factor did not sufficiently control for other known risk factors; thus, how uric acid causes hypertension is not fully understood [10].

To decipher the relationship between SUA and hypertension while controlling for known risk factors, including sex, BMI, eGFR, and several metabolic factors, we collected a dynamic cohort comprising primarily senior citizens (the Tianjin General Hospital cohort) in the city of Tianjin, China, and performed physical exams and clinical/biochemical tests each year from 2006 to 2011.

\section{Methods}

\section{Subjects}

We recruited 7032 subjects for this study from 2006 to 2011. Recruited subjects were asked to participate in an annual physical examination up to 2011. At their entry examinations, a total of 3441 subjects were found to have hypertension, and during the follow-up period, 744 out of the remaining 3591 subjects developed incident hypertension. We organized two studies: (1) a cross-sectional survey using the data at the study entry; and (2) a dynamic cohort study of the subjects who did not have hypertension at study entry. Subject recruiting protocols were reviewed and approved by the Human Ethics Committee of Tianjin Medical University. Subjects gave informed consent prior to participating in this study. All experiments were performed in accordance with relevant guidelines and regulations.

All of the subjects who were randomly assigned were eligible for this study provided they met all following criteria at the baseline: (1) no evidence of hypertension (defined as systolic blood pressure of $\geq 140 \mathrm{mmHg}$ and/ or a diastolic blood pressure of $\geq 90 \mathrm{mmHg}$, and/or the current use of antihypertensive medication, regardless of the recorded blood pressure); (2) no evidence of left ventricular hypertrophy/myocardial infarction/heart failure; (3) no evidence of diabetes mellitus (defined as a fasting plasma glucose $\geq 126 \mathrm{mg} / \mathrm{dl}(7.0 \mathrm{mmol} / \mathrm{L})$, or the use of antidiabetic medications); (4) no evidence of kidney disease or gout, and no use of diuretics/allopurinol to lower uric acid levels; (5) availability of serum uric acid measurement at baseline or the follow-up examinations; and (6) availability of blood pressure at baseline or follow-up examinations.

\section{Annual physical exams}

At each exam, all subjects were measured twice for height and weight (using identical standardized anthropometric scales), and body mass index (BMI) was calculated as body weight $(\mathrm{kg})$ divided by height squared $\left(\mathrm{m}^{2}\right)$. A well-trained nurse or doctor measured resting blood pressure once using a standard mercury sphygmomanometer with the subject in the sitting position after at least $5 \mathrm{~min}$ of rest. All the subjects fasted overnight for at least $12 \mathrm{~h}$ before blood sampling. Venous blood samples were obtained from the antecubital vein for measuring SUA levels (Additional file 1: Tables S1 and S2), fasting glucose, lipid profiles (total cholesterol, triglycerides), renal function (plasma creatinine, blood urea nitrogen), liver function (total serum protein, albumin, globulin, total and direct bilirubin, and aspartate aminotransferase), white cell count, hemoglobin tests. SUA levels were measured by enzymatic methods (Chemistry Analyzer Au2700, Olympus Medical Engineering Company, Japan). Newly diagnosed diseases during the past year and identified at the physical exam were documented.

\section{Definitions}

Hypertension was defined as a systolic blood pressure of $\geq 140 \mathrm{mmHg}$ and/or a diastolic blood pressure of $\geq 90 \mathrm{mmHg}$, and/or the current use of antihypertensive medication. For calculating incidence rates, we used the first occasion of documented hypertension per subject as the index event. According to the recommended criteria for the Chinese people [11], normal weight was defined as a BMI of $18.5-23.9 \mathrm{~kg} / \mathrm{m}^{2}$, overweight as a BMI of $24.0-27.9 \mathrm{~kg} / \mathrm{m}^{2}$, and obesity as a BMI $\geq 28 \mathrm{~kg} /$ $\mathrm{m}^{2}$. Values of estimated glomerular filtration rate (eGFR; $\mathrm{ml} / \mathrm{min} / 1.73 \mathrm{~m}^{2}$ ) were calculated using the equation proposed by investigators in the Chronic Kidney Disease Epidemiology Collaboration. Hyperuricemia was defined as SUA $\geq 7.0 \mathrm{mg} / \mathrm{dL}(420 \mu \mathrm{mol} / \mathrm{L})$ in males and $\geq 6.0 \mathrm{mg} / \mathrm{dL}(360 \mu \mathrm{mol} / \mathrm{L})$ in females. SUA levels were 
categorized by quartiles as $<4.69,4.69-5.58,5.58-6.52$, and $\geq 6.52 \mathrm{mg} / \mathrm{dl}$.

In this paper, the data are divided into three groups: (1) baseline data from subjects who did not have hypertension at the study entry; (2) follow-up means calculated from specified observations starting from the time of the baseline visit through the last follow-up visit or first occasion of hypertension (index event); and (3) normal-eGFR subjects with eGFR $\geq 60 \mathrm{ml} / \mathrm{min} / 1.73 \mathrm{~m}^{2}$ (chronic kidney disease was defined as eGFR $<60 \mathrm{ml} / \mathrm{min} / 1.73 \mathrm{~m}^{2}$ ) [12].

\section{Data analysis}

The basic characteristics of the sample are described by descriptive statistics. The data for continuous variables are reported as the mean $\pm \mathrm{SD}$ and median (interquartile range), and the data for categorical variables are reported as percentages (\%). The clinical characteristics between nonhypertensive and hypertensive groups were analyzed using the Student's $t$ test for data that were normally distributed, and the MannWhitney U-test for non-normal distributions, The

Table 1 Characteristics of subjects [mean \pm SD or $N(\%)$ ] during baseline and follow-up periods ${ }^{a}$ by status at follow-up (hypertensive, nonhypertensive)

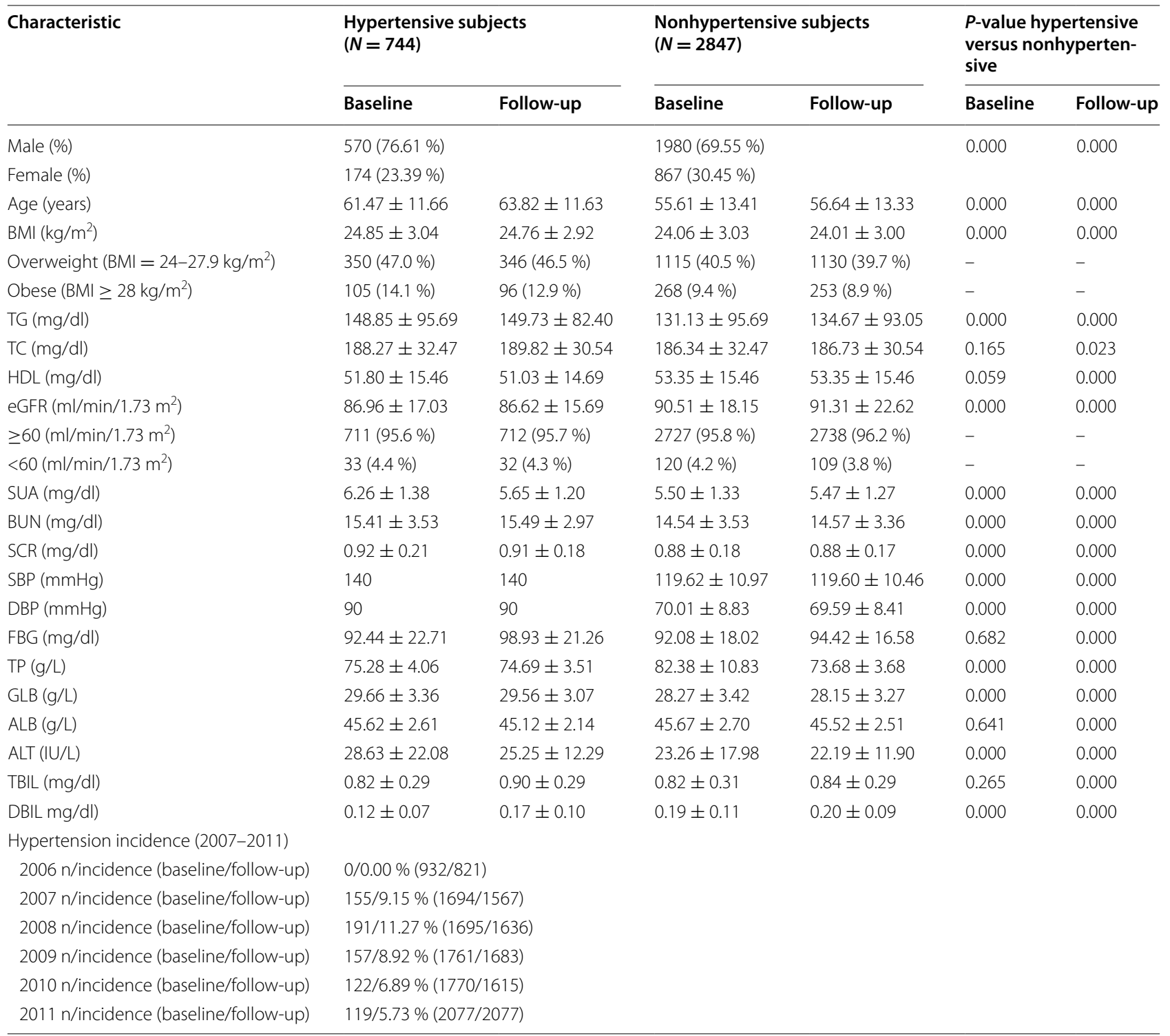

BMI body mass index; TG plasma levels of triglycerides; TC total cholesterol; HDL high-density lipoprotein; eGFR estimated glomerular filtration rate; SUA serum uric acid; $B U N$ blood urea nitrogen; $S C R$ serum creatinine; $S B P$ systolic blood pressure; $D B P$ diastolic blood pressure; $F B G$ fasting plasma glucose; $T P$ plasma total protein; GLB globulin; $A L B$ albumin; $A L T$ alanine aminotransferase; TBIL total bilirubin; DBIL direct bilirubin

a Means during the follow-up period are specified observations starting from the time of the baseline visit through the last visit or incidence of hypertension 
Chi squared test was used to compare differences in percentages in the dichotomous variables. A Cox proportional hazards model was used to assess the relationship between SUA and hypertension; hazard ratios and $95 \%$ confidence intervals (CIs) for hypertension were calculated. Kaplan-Meier survival analysis was used to compare incidence of hypertension among individuals with each SUA quartile. The log rank test was used to compare the curves. Receiver operating characteristic (ROC) curves were generated to obtain the area under the curve (AUC) as a prediction of hypertension from SUA levels. Status for hypertension, myocardial infarction, stroke, type 1 and type 2 diabetes, and 45 other diseases was documented in a Filemaker Pro database. For statistical inference, a bilateral $P$-value of $<0.05$ was considered statistically significant. All statistical analyses were carried out using SPSS statistical software, version 17.0 (SPSS Inc., Chicago, IL, USA) for Windows.

\section{Results}

In our dynamic cohort study, 3591 individuals were included; the mean age of the subjects was $56.83 \pm 13.28$ years, with 2550 males $(71.0 \%$; mean \pm SD, $59.82 \pm 13.30$ years of age) and 1041 females (29.0; $57.27 \pm 13.02$ years of age). The characteristics of the subjects at baseline and during the follow-up period are shown in Tables 1 and 2. BMI, age, most blood measures, and the prevalence of male sex and obesity increased in the hypertensive group, but eGFR and direct bilirubin levels significantly decreased. After 6 years, cumulative prevalence of hypertension was $20.7 \%$ (22.4 \% in males, $16.7 \%$ in females; $\left.X^{2}=14.307, P<0.001\right)$. The prevalence of hyperuricemia was $17.5 \%$ (19.9\% in males, $11.7 \%$ in females; $\left.X^{2}=33.942, P<0.001\right)$. A cross-sectional survey was organized using baseline data from 3591 subjects enrolled. Logistic regression analysis identified SUA level as a factor that increased hypertension risk (Table 3). In an unadjusted model odds ratio (95\% CIs) for hypertension, compared with the lowest SUA quartile $(<4.69 \mathrm{mg} /$ $\mathrm{dl}$ ), the $4.69-5.58,5.58-6.52$, and $\geq 6.52 \mathrm{mg} / \mathrm{dl}$ quartiles yielded odds ratios (95\% confidence intervals) for hypertension of 1.989 (1.497-2.644), 2.975 (2.267-3.905), and 4.684 (3.596-6.102), respectively. After controlling for confounders, in adjusted models (models 2 and 3 ) the difference remained, and risk of hypertension was higher as SUA levels increased.

We conduct a dynamic cohort study of the subjects who did not have hypertension at the study entry. A Cox proportional hazards model was used to assess the relationship between SUA and hypertension. At baseline, in an unadjusted model (Table 4, model 1) the hazard ratios (95\% CIs) for hypertension in the second, third, and fourth SUA quartiles, compared with the first SUA quartile, were 1.652 (95\% CI 1.265-2.156), 2.195 (95\% CI 1.705-2.825), and 3.058 (95\% CI 2.399-3.899), respectively; after adjusting for age and sex (model 2), they were 1.644 (95\% CI 1.253-2.158), 2.274 (95\% CI 1.746-2.963), and 3.174 (95\% CI 2.448-4.114), respectively. The association was also significant after further adjustment for multiple metabolic parameters (model 3): the hazard ratios comparing the second, third, and fourth SUA quartiles versus first SUA quartile were 1.745 (95\% CI 1.321-2.328), 2.349 (95 \% CI 1.774-3.111), and 3.152 (95 \% CI 2.371-4.191), respectively $(P<0.001)$. When the analyses were repeated using SUA levels as a continuous variable, significant associations were observed in models $1-3$ between increased SUA levels $(1 \mu \mathrm{mol} / \mathrm{L})$ and the risk of hypertension.

When we used means for subject characteristics during the follow-up period, in unadjusted models (Table 4, model 4), significant differences were identified in the second and fourth SUA quartile, but not the third quartile, compared with the first. After controlling for confounders, in adjusted models (models 5 and 6) the difference remained, and risk of hypertension was higher in the second and fourth SUA quartiles.

Table 2 Correlation analyses among serum uric acid levels and clinical characteristics during baseline and follow-up periods

\begin{tabular}{|c|c|c|c|c|}
\hline \multirow[t]{2}{*}{ Characteristic } & \multicolumn{2}{|c|}{ Baseline } & \multicolumn{2}{|c|}{ Follow-up } \\
\hline & $R$ & $P$ & $r$ & $P$ \\
\hline Age (years) & 0.044 & 0.009 & 0.024 & 0.151 \\
\hline BMI $\left(\mathrm{kg} / \mathrm{m}^{2}\right)$ & 0.340 & 0.000 & 0.362 & 0.000 \\
\hline TG (mg/dl) & 0.262 & 0.000 & 0.297 & 0.000 \\
\hline TC (mg/dl) & 0.023 & 0.278 & 0.013 & 0.509 \\
\hline $\mathrm{HDL}(\mathrm{mg} / \mathrm{dl})$ & -0.232 & 0.000 & -0.311 & 0.000 \\
\hline $\operatorname{eGFR}\left(\mathrm{ml} / \mathrm{min} / 1.73 \mathrm{~m}^{2}\right)$ & -0.236 & 0.000 & -0.195 & 0.000 \\
\hline BUN (mg/dl) & 0.192 & 0.000 & 0.222 & 0.000 \\
\hline $\mathrm{SCR}(\mathrm{mg} / \mathrm{dl})$ & 0.435 & 0.000 & 0.485 & 0.000 \\
\hline SBP $(\mathrm{mmHg})$ & 0.171 & 0.000 & 0.165 & 0.000 \\
\hline $\mathrm{DBP}(\mathrm{mmHg})$ & 0.189 & 0.000 & 0.201 & 0.000 \\
\hline FBG (mg/dl) & 0.023 & 0.162 & 0.046 & 0.006 \\
\hline $\mathrm{TP}(\mathrm{g} / \mathrm{L})$ & -0.092 & 0.000 & 0.088 & 0.000 \\
\hline GLB (g/L) & 0.056 & 0.001 & 0.023 & 0.175 \\
\hline ALB (g/L) & 0.080 & 0.000 & 0.105 & 0.000 \\
\hline ALT (IU/L) & 0.136 & 0.000 & 0.223 & 0.000 \\
\hline TBIL (mg/dl) & 0.140 & 0.000 & 0.159 & 0.000 \\
\hline DBIL (mg/dl) & -0.022 & 0.192 & 0.067 & 0.000 \\
\hline
\end{tabular}

$B M I$ body mass index; TG plasma levels of triglycerides; TC total cholesterol; $H D L$ high-density lipoprotein; eGFR estimated glomerular filtration rate; SUA serum uric acid; $B U N$ blood urea nitrogen; $S C R$ serum creatinine; $S B P$ systolic blood pressure; $D B P$ diastolic blood pressure; $F B G$ fasting plasma glucose; TP plasma total protein; GLB globulin; $A L B$ albumin; $A L T$ alanine aminotransferase; TBIL total bilirubin; $D B I L$ direct bilirubin 
Table 3 Odds ratio of SUA levels for incidence hypertension in a cross sectional survey of 3591 subjects

\begin{tabular}{|c|c|c|c|c|}
\hline Model & $N(\%)$ & Odds ratio & $95 \% \mathrm{Cl}$ & $P$-value \\
\hline \multicolumn{5}{|c|}{ Model 1: unadjusted baseline values of variables } \\
\hline Quartile 1 & $903(25.1 \%)$ & - & - & 0.000 \\
\hline Quartile 2 & $886(24.7 \%)$ & 1.989 & $1.497-2.644$ & 0.000 \\
\hline Quartile 3 & $905(25.2 \%)$ & 2.975 & $2.267-3.905$ & 0.000 \\
\hline Quartile 4 & $897(25.0 \%)$ & 4.684 & $3.596-6.102$ & 0.000 \\
\hline SUA as a continuous variable $(\mu \mathrm{mol} / L)$ & 3591 & 1.252 & $1.103-1.454$ & 0.000 \\
\hline \multicolumn{5}{|c|}{ Model 2: model 1 adjusted for age and gender } \\
\hline Quartile 1 & - & - & - & 0.000 \\
\hline Quartile 2 & - & 2.107 & $1.568-2.833$ & 0.000 \\
\hline Quartile 3 & - & 3.432 & $2.558-4.604$ & 0.000 \\
\hline Quartile 4 & - & 5.492 & $4.091-7.372$ & 0.000 \\
\hline SUA as a continuous variable ( $\mu \mathrm{mol} / \mathrm{L})$ & - & 1.298 & $1.047-1.609$ & 0.017 \\
\hline \multicolumn{5}{|c|}{ Model 3: model 2 further adjusted for other confounders } \\
\hline Quartile 1 & - & - & - & 0.000 \\
\hline Quartile 2 & - & 2.141 & $1.534-2.987$ & 0.000 \\
\hline Quartile 3 & - & 3.213 & $2.288-4.511$ & 0.000 \\
\hline Quartile 4 & - & 5.624 & $3.936-8.037$ & 0.000 \\
\hline SUA as a continuous variable ( $\mu \mathrm{mol} / \mathrm{L})$ & - & 1.686 & $1.245-2.283$ & 0.001 \\
\hline
\end{tabular}

Quartiles based on serum uric acid levels: $1,<4.69 \mathrm{mg} / \mathrm{dl} ; 2,4.69-5.58 \mathrm{mg} / \mathrm{dl} ; 3,5.58-6.52 \mathrm{mg} / \mathrm{dl} ; 4, \geq 6.52 \mathrm{mg} / \mathrm{dl}$

Model 3 was further adjusted for BMI, triglycerides, eGFR, blood urea nitrogen, serum creatinine, plasma total protein, globulin, alanine aminotransferase, and direct bilirubin

$P<0.05$ was considered statistically significant

To investigate effects of impaired renal function on uric acid phenotype correlations, we divided our cohort by normal $\left(\geq 60 \mathrm{ml} / \mathrm{min} / 1.73 \mathrm{~m}^{2}\right)$ and abnormal $(<60 \mathrm{ml} /$ $\mathrm{min} / 1.73 \mathrm{~m}^{2}$ ) eGFR. The Cox regression results in normal-eGFR subjects were relatively unchanged (Table 5). In an unadjusted model (model A), the hazard ratios (95\% CIs) for hypertension in the second, third, and fourth SUA quartiles, compared with the first SUA quartile, were 1.738 (95 \% CI 1.321-2.287), 2.166 (95\% CI 1.666-2.815), and 3.065 (95\% CI 2.386-3.937), respectively. After adjusting for age and sex (model B), they were 1.761 (95 \% CI 1.331-2.329), 2.305 (95 \% CI 1.7513.035 ), and 3.320 (95\% CI 2.535-4.347), respectively. After further adjustment for multiple metabolic parameters (model C), they were 1.662 (95 \% CI 1.261-2.190), 2.028 (95 \% CI 1.552-2.649), and 2.747 (95 \% CI 2.115$3.566)$, respectively $(P<0.001)$.

Survival analyses for hypertension status in uric acid quartiles during the 6-year period (2006-2011) showed significant dose effects in each quartile. A Kaplan-Meier survival analysis showed that hyperuricemia predicted higher incidences of hypertension in a dose-dependent manner: hypertension onsets significantly differed across SUA quartiles (Fig. 1a, b).

Because SUA level is considered as an index of renal function, we divided subjects by eGFR and reexamined the correlations. We found that most correlations between SUA and hypertension and liver function phenotypes remained essentially unchanged in the normaleGFR group (Table 5; Fig. 1c).

Receiver operating characteristic curves were generated to obtain the AUC as a prediction of hypertension from SUA levels. ROC analysis revealed an AUC of 0.659 in the ability of SUA levels to predict hypertension diagnosis in the total data. This model was also found to be significantly different than the random predictor (AUC $=0.500$, $P<0.001$ ). The sensitivity and specificity were 65.1 and $58.8 \%$, respectively. For men, the sensitivity and specificity were 71.6 and $49.7 \%$, respectively, and the AUC was 0.647 . For women, the sensitivity and specificity were 70.1 and $59.8 \%$, respectively, and the AUC was 0.683 (Fig. 2a-c).

\section{Discussion}

In a Chinese senior dynamic cohort (the Tianjin General Hospital cohort), we found a significant association between higher SUA concentrations and an increased hazard of incident hypertension. This association was independent of age, sex, BMI, triglycerides, eGFR, blood urea nitrogen, serum creatinine, plasma total protein, globulin, alanine aminotransferase, and direct bilirubin. Using SUA measurements as a continuous measure or a dichotomous variable did not change 
Table 4 Hazard ratios of SUA levels for incidence hypertension during 6 years of follow-up among 3591 subjects without hypertension at the entyry examination

\begin{tabular}{|c|c|c|c|c|}
\hline Model & $N(\%)$ & Hazard ratio & $95 \% \mathrm{Cl}$ & $P$-value \\
\hline \multicolumn{5}{|l|}{ Baseline values: models 1-3 } \\
\hline \multicolumn{5}{|c|}{ Model 1: unadjusted baseline values of variables } \\
\hline Quartile 1 & $903(25.1 \%)$ & - & - & 0.000 \\
\hline Quartile 2 & $886(24.7 \%)$ & 1.652 & $1.265-2.156$ & 0.000 \\
\hline Quartile 3 & $905(25.2 \%)$ & 2.195 & $1.705-2.825$ & 0.000 \\
\hline Quartile 4 & $897(25.0 \%)$ & 3.058 & $2.399-3.899$ & 0.000 \\
\hline SUA as a continuous variable ( $\mu \mathrm{mol} / \mathrm{L})$ & 3591 & 2.039 & $1.747-2.379$ & 0.000 \\
\hline \multicolumn{5}{|c|}{ Model 2: model 1 adjusted for age and gender } \\
\hline Quartile 1 & - & - & - & 0.000 \\
\hline Quartile 2 & - & 1.644 & $1.253-2.158$ & 0.000 \\
\hline Quartile 3 & - & 2.274 & $1.746-2.963$ & 0.000 \\
\hline Quartile 4 & - & 3.174 & $2.448-4.114$ & 0.000 \\
\hline SUA as a continuous variable ( $\mu \mathrm{mol} / \mathrm{L})$ & - & 1.703 & $1.292-2.243$ & 0.000 \\
\hline \multicolumn{5}{|c|}{ Model 3: model 2 further adjusted for other confounders } \\
\hline Quartile 1 & - & - & - & 0.000 \\
\hline Quartile 2 & - & 1.754 & $1.321-2.328$ & 0.000 \\
\hline Quartile 3 & - & 2.349 & $1.774-3.111$ & 0.000 \\
\hline Quartile 4 & - & 3.152 & $2.371-4.191$ & 0.000 \\
\hline SUA as a continuous variable ( $\mu \mathrm{mol} / \mathrm{L})$ & - & 1.793 & $1.512-2.126$ & 0.000 \\
\hline \multicolumn{5}{|c|}{ Follow-up values: models 4-6 } \\
\hline \multicolumn{5}{|c|}{ Model 4: unadjusted follow-up values of variables } \\
\hline Quartile 1 & $897(25.0 \%)$ & - & - & 0.018 \\
\hline Quartile 2 & $878(24.5 \%)$ & 1.279 & $1.029-1.590$ & 0.027 \\
\hline Quartile 3 & $917(25.5 \%)$ & 1.160 & $0.886-1.519$ & 0.281 \\
\hline Quartile 4 & $899(25.0 \%)$ & 1.366 & $1.120-1.666$ & $0.002^{*}$ \\
\hline SUA as a continuous variable ( $\mu \mathrm{mol} / \mathrm{L})$ & 3591 & 1.104 & $1.030-1.184$ & $0.005^{*}$ \\
\hline \multicolumn{5}{|l|}{ Model 5: model 4 adjusted for age and sex } \\
\hline Quartile 1 & - & - & - & $0.009^{*}$ \\
\hline Quartile 2 & - & 1.243 & $1.060-1.639$ & $0.013^{*}$ \\
\hline Quartile 3 & - & 1.410 & $0.947-1.630$ & 0.117 \\
\hline Quartile 4 & - & 1.264 & $1.155-1.722$ & $0.001^{*}$ \\
\hline SUA as a continuous variable ( $\mu \mathrm{mol} / \mathrm{L})$ & - & 1.264 & $1.198-1.335$ & 0.000 \\
\hline \multicolumn{5}{|c|}{ Model 6: model 5 further adjusted for other confounders } \\
\hline Quartile 1 & - & - & - & $0.026^{*}$ \\
\hline Quartile 2 & - & 1.103 & $1.031-1.179$ & $0.004^{*}$ \\
\hline Quartile 3 & - & 1.043 & $0.778-1.398$ & 0.780 \\
\hline Quartile 4 & - & 1.350 & $1.106-1.648$ & $0.003^{*}$ \\
\hline SUA as a continuous variable ( $\mu \mathrm{mol} / \mathrm{L})$ & - & 1.157 & $1.066-1.255$ & $0.001^{*}$ \\
\hline
\end{tabular}

Model 1-3 (Baseline) Quartiles based on serum uric acid levels: 1, $<4.69 \mathrm{mg} / \mathrm{dl} ; 2,4.69-5.58 \mathrm{mg} / \mathrm{dl} ; 3,5.58-6.52 \mathrm{mg} / \mathrm{dl} ; 4, \geq 6.52 \mathrm{mg} / \mathrm{dl}$

Model 4-6 (Means during follow-up period) Quartiles based on serum uric acid levels: $1,<4.64 \mathrm{mg} / \mathrm{dl} ; 2,4.64-5.41 \mathrm{mg} / \mathrm{dl} ; 3,5.41-5.78 \mathrm{mg} / \mathrm{dl} ; 4, \geq 5.78 \mathrm{mg} / \mathrm{dl}$

Model 3 was further adjusted for BMI, triglycerides, eGFR, blood urea nitrogen, serum creatinine, plasma total protein, globulin, alanine aminotransferase, and direct bilirubin

Model 6 was further adjusted for BMI, triglycerides, total cholesterol, high-density lipoproteins, estimated glomerular filtration rate, blood urea nitrogen, serum creatinine, fasting plasma glucose, plasma total protein, globulin, albumin, alanine aminotransferase, total bilirubin, and direct bilirubin

* $P<0.05$ was considered statistically significant

our findings. There were consistent findings across the various adjusted and unadjusted analyses. These results demonstrate that SUA level is a durable marker of risk for hypertension. The relative risk of hypertension increased in a dose-dependent manner with increasing uric acid quartiles. 
Table 5 Incidence of hypertension by serum uric acid quartile in normal-eGFR subjects

\begin{tabular}{|c|c|c|c|c|}
\hline Model & $N(\%)$ & Hazard ratio & $95 \% \mathrm{Cl}$ & $P$-value \\
\hline \multicolumn{5}{|l|}{ Model A: unadjusted } \\
\hline Quartile 1 & $844(24.5 \%)$ & - & - & 0.000 \\
\hline Quartile 2 & $872(25.4 \%)$ & 1.738 & $1.321-2.287$ & 0.000 \\
\hline Quartile 3 & $855(24.9 \%)$ & 2.166 & $1.666-2.815$ & 0.000 \\
\hline Quartile 4 & $867(25.2 \%)$ & 3.065 & $2.386-3.937$ & 0.000 \\
\hline SUA as a continuous variable ( $\mu \mathrm{mol} / \mathrm{L})$ & 3438 & 2.061 & $1.758-2.416$ & 0.000 \\
\hline \multicolumn{5}{|l|}{ Model B: model A adjusted for age and sex } \\
\hline Quartile 1 & - & - & - & 0.000 \\
\hline Quartile 2 & - & 1.761 & $1.331-2.329$ & 0.000 \\
\hline Quartile 3 & - & 2.305 & $1.751-3.035$ & 0.000 \\
\hline Quartile 4 & - & 3.320 & $2.535-4.347$ & 0.000 \\
\hline SUA as a continuous variable ( $\mu \mathrm{mol} / \mathrm{L})$ & - & 2.040 & $1.739-2.392$ & 0.000 \\
\hline \multicolumn{5}{|c|}{ Model C: model B further adjusted for other confounders } \\
\hline Quartile 1 & - & - & - & 0.000 \\
\hline Quartile 2 & - & 1.662 & $1.261-2.190$ & 0.000 \\
\hline Quartile 3 & - & 2.028 & $1.552-2.649$ & 0.000 \\
\hline Quartile 4 & - & 2.747 & $2.115-3.566$ & 0.000 \\
\hline SUA as a continuous variable ( $\mu \mathrm{mol} / \mathrm{L})$ & - & 1.807 & $1.519-2.150$ & 0.000 \\
\hline
\end{tabular}

Model C was further adjusted for BMI, TG, eGFR, blood urea nitrogen, serum creatinine, plasma total protein, globulin, alanine aminotransferase, and direct bilirubin Quartiles based on serum uric acid levels: $1,<4.66 \mathrm{mg} / \mathrm{dl} ; 2,4.66-5.53 \mathrm{mg} / \mathrm{dl} ; 3,5.53-6.46 \mathrm{mg} / \mathrm{dl} ; 4, \geq 6.46 \mathrm{mg} / \mathrm{dl}$

Our findings relating SUA to hypertension incidence with short-term follow-up (up to 5 years) confirm several previous reports. Our results are similar to observations in cross-sectional analyses and longitudinal studies [5-7], and several studies report linear hazard ratios comparable to ours [8-14]. In the Multiple Risk Factor Intervention Trial (MRFIT) study [8] and the Beaver Dam study [9] the hazard ratios for hypertension were 1.02 and 1.31, respectively. Our reported hazard ratios are larger than those in the MRFIT study but smaller than in the Beaver Dam study. In our cohort, the cumulative prevalence of hypertension was $20.7 \%$ (22.4\% in men, $16.7 \%$ in women). Zhang et al. reported prevalences of hypertension as $19.0 \%$ in men and $11.0 \%$ in women in a Chinese community [14], and Sundström et al. [15] reported a hypertension prevalence of $13.8 \%$. Compared with these previous studies, our cohort had a high cumulative incidence of hypertension, and the cause of this difference might be our older study population. The prevalence of hypertension was somewhat different from the general population in China. Qi et al. [16] reported that the adjusted prevalence of hypertension was $20.9 \%$, which is very close to ours, but Yang et al. [17] reported a higher prevalence of $30.8 \%$ in northeastern China by a cross-sectional study.

The survival analysis showed that hyperuricemia predicted higher incidences of hypertension in a dosedependent manner: hypertension onset significantly differed across SUA quartiles, a result consistent with that of Masuo et al. [18]. Despite higher hazard ratios in the top quartile for SUA, in our dynamic cohort the SUA levels dropped across all quartiles over the follow-up period. These results are somewhat contradictory to previous longitudinal studies $[9,10]$. The disparity may be due to three main reasons. First, in the present study, we did not assess lifestyle-related variables such as smoking, alcohol, physical training, and eating habits, which may have affected SUA levels during the follow-up period. Second, there is known variation in SUA level when measured repeatedly, and because several drugs that may also have affected SUA levels were not assessed, we could not pursue this question. The last reason may result from our dynamic cohort: as new, younger subjects joined the cohort and older subjects were lost to follow-up, our results may be partially due to the younger age of more recent subjects.

Our study had strengths and limitations. A strength of our study is that the cross-sectional study and the dynamic cohort study generated consistent results. However, one limitation was that we did not collect data on lifestyle factors and socioeconomic status, which are known to vary in risk of hypertension [19, 20]. Despite this, the association between SUA and hypertension is unlikely mediated by these factors, and undiagnosed high SUA is less likely to cause changes in lifestyle and socioeconomic status. Another limitation was that the subjects were "healthy persons" who had routine health examinations but were not patients who sought medical care. 

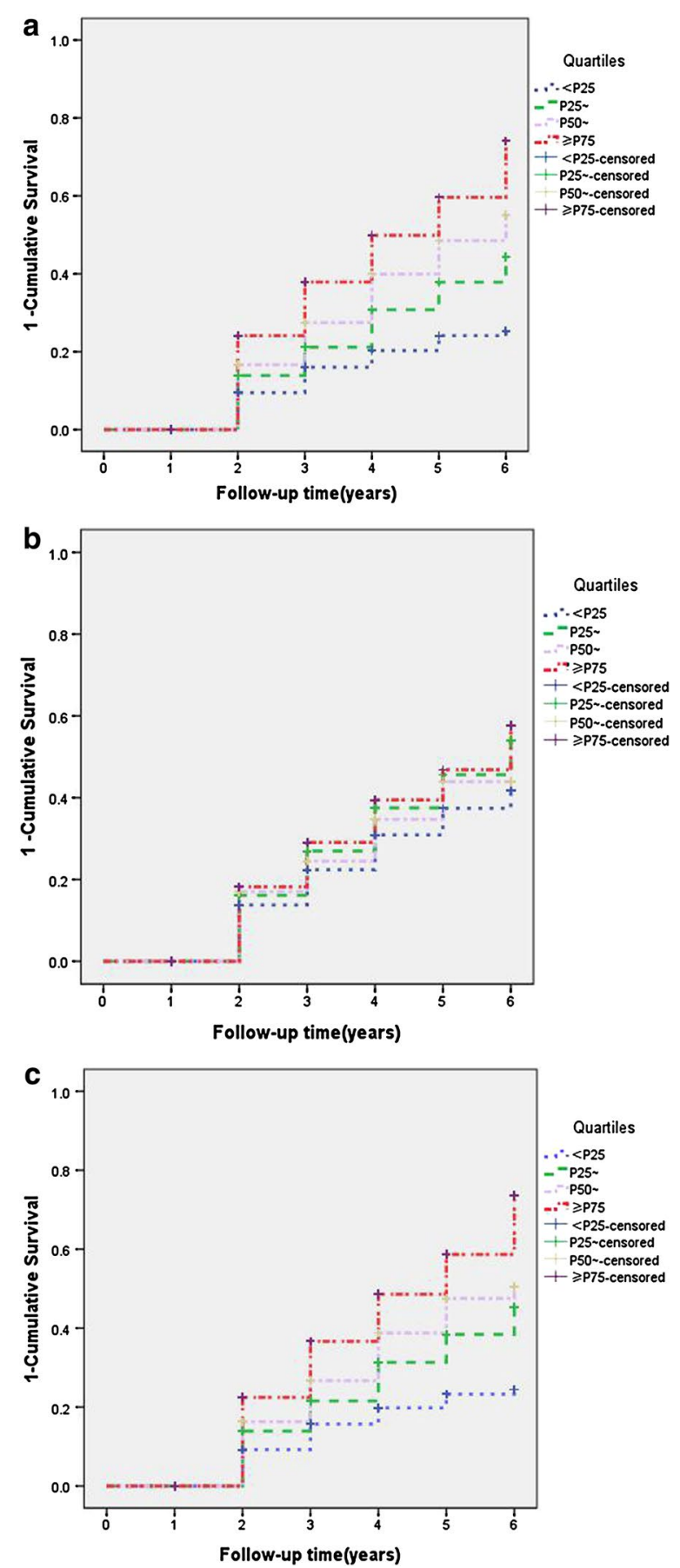

Fig. 1 Kaplan-Meier curves for hypertension among quartiles of serum uric acid (SUA) levels. Baseline quartiles of SUA were defined as follows: first quartile, $<4.69 \mathrm{mg} / \mathrm{dl}\left(<\mathrm{P}_{25}\right)$; second quartile, $4.69-5.58 \mathrm{mg} / \mathrm{dl}\left(\mathrm{P}_{25} \sim\right)$; third quartile, 5.58-6.52 mg/dl $\left(\mathrm{P}_{50} \sim\right)$; and fourth quartile, $\geq 6.52 \mathrm{mg} / \mathrm{dl}\left(\geq P_{75}\right)$. a Baseline, all subjects (log-rank test $P$-value $<0.01,<P_{25}$ vs. other quartiles). b Follow-up, all subjects (log-rank test $P$-value $<0.05,<P_{25}$ vs. other quartiles). c Baseline, normal-eGFR subjects only (log-rank test $P$-value $<0.01,<P_{25}$ vs. other quartiles)
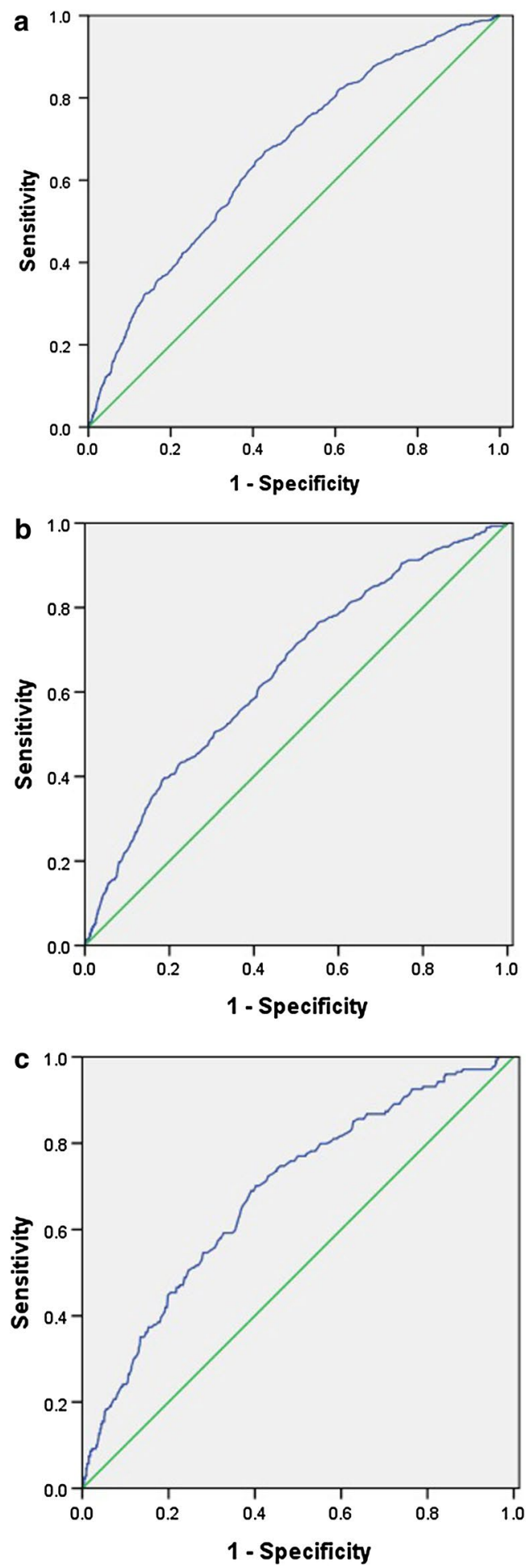

Fig. 2 Receiver operating characteristic curve for the prediction of hypertension from serum uric acid levels in total data [a; area under the curve $(A \cup C)=0.659$ ], in men $(\mathbf{b}$; $A \cup C=0.647)$, and in women (c; $A \cup C=0.683)$ 
The hospital was a top tertiary care center, and persons who had annual health examinations at this hospital were likely to civil servants, university teachers, and employees of government-funded agencies-persons of high socioeconomic status. Thus, they are not representative of the general population. Further replication our findings in general population is warranted, especially among subjects with low socioeconomic status.

\section{Conclusions}

We found that SUA levels predicted incident hypertension in a Chinese cohort of senior persons. Increased SUA levels were significantly and independently associated with the incidence of hypertension over a 6-year period in a dynamic cohort without hypertension at baseline. A survival analysis showed that elevated SUA levels predicted higher cumulative incidence of hypertension. To prevent cardiovascular events, this relationship between increased SUA levels and increased incidence of hypertension should be noted. In future research, we plan to investigate clinical outcomes of individuals with elevated SUA levels in our cohort and test genetic backgrounds related to hyperuricemia in general populations.

\section{Additional file}

Additional file 1. Supplement Tables S1 and S2.

\section{Abbreviations \\ SUA: serum uric acid; BMI: body mass index; eGFR: estimated glomerular filtration rate; $\mathrm{Cl}$ : confidence interval; SD: standard deviation; ROC: receiver operating characteristic; AUC: area under the curve; MRFIT: multiple risk factor intervention trial.}

\section{Authors' contributions}

$W D L, X Y$, and $Y W$ conceived and designed the study; $W D L, X Y$, and FW wrote the manuscript; FW, NS, CC, SF, JT, WX, and WS, performed the experiments; FW, WS, CC, SF, and WDL analyzed the data; NS, CC, WX, SF, JT, and WS collected subjects and clinical data. All authors have reviewed the manuscript. All authors read and approved the final manuscript.

\section{Author details \\ ${ }^{1}$ Research Center of Basic Medical Sciences, Tianjin Medical University, 22 Qixiangtai Road, Tianjin 300070, People's Republic of China. ${ }^{2}$ Tianjin General Hospital, Tianjin Medical University, 154 Anshan Road, Tianjin 300052, People's Republic of China. ${ }^{3}$ Aging Research Center, Department of Neurobiology, Care Sciences and Society (NVS), Karolinska Institute, Stockholm, Sweden. ${ }^{4}$ School of Public Health, Tianjin Medical University, 22 Qixiangtai Road, Tianjin 300070, People's Republic of China. ${ }^{5}$ Department of Epidemiology and Biostatistics, School of Public Health, Tianjin Medical University, 22 Qixiangtai Road, Tian- jin 300070, People's Republic of China.}

\section{Acknowledgements \\ We thank all subjects who took part in this study.}

\section{Competing interests}

The authors declare that they have no competing interests.

\section{Sources of funding}

This work was supported in part by Grant 81070576 from the National Natural Science Foundation of China and grant 12JCZDJC24700 from the
Tianjin Municipal Science and Technology Commission to WDL, and by Grant 15ZCZDSY00500 from Tianjin Key Project of Scientific Support to YW.

Received: 15 December 2015 Accepted: 12 April 2016

Published online: 30 April 2016

\section{References}

1. Fields LE, Burt VL, Cutler JA, Hughes J, Roccella EJ, Sorlie P. The burden of adult hypertension in the United States 1999 to 2000: a rising tide. Hypertension. 2004;44:398-404.

2. Grayson PC, Kim SY, LaValley M, Choi HK. Hyperuricemia and incident hypertension: a systematic review and meta-analysis. Arthritis Care Res (Hoboken). 2011;63:102-10.

3. Chen JH, Chuang SY, Chen HJ, Yeh WT, Pan WH. Serum uric acid level as an independent risk factor for all-cause, cardiovascular, and ischemic stroke mortality: a Chinese cohort study. Arthritis Rheum. 2009;61:225-32.

4. Cai Z, Xu X, Wu X, Zhou C, Li D. Hyperuricemia and the metabolic syndrome in Hangzhou. Asia Pac J Clin Nutr. 2009;18:81-7.

5. Perlstein TS, Gumieniak O, Williams GH, Sparrow D, Vokonas PS, Gaziano $M$, et al. Uric acid and the development of hypertension: the normative aging study. Hypertension. 2006;48:1031-6.

6. Nagahama K, Inoue T, Iseki K, Touma T, Kinjo K, Ohya Y, et al. Hyperuricemia as a predictor of hypertension in a screened cohort in Okinawa Japan. Hypertens Res. 2004;27:835-41.

7. Mellen PB, Bleyer AJ, Erlinger TP, Evans GW, Nieto FJ, Wagenknecht $L E$, et al. Serum uric acid predicts incident hypertension in a biethnic cohort: the atherosclerosis risk in communities study. Hypertension. 2006:48:1037-42.

8. Krishnan E, Kwoh CK, Schumacher HR, Kuller L. Hyperuricemia and incidence of hypertension among men without metabolic syndrome. Hypertension. 2007;49:298-303.

9. Shankar A, Klein R, Klein BE, Nieto FJ. The association between serum uric acid level and long-term incidence of hypertension: population-based cohort study. J Hum Hypertens. 2006;20:937-45.

10. Wang J, Qin T, Chen J, Li Y, Wang L, Huang H, et al. Hyperuricemia and risk of incident hypertension: a systematic review and meta-analysis of observational studies. PLoS One. 2014;9:e114259.

11. Wu Y. Overweight and obesity in China. BMJ. 2006;333:362-3.

12. Levey AS, Stevens LA, Schmid CH, Zhang YL, Castro AF 3rd, Feldman HI, et al. A new equation to estimate glomerular filtration rate. Ann Int Med. 2009;150:604-12.

13. Gaffo AL, Jacobs DR Jr, Sijtsma F, Lewis CE, Mikuls TR, Saag KG. Serum urate association with hypertension in young adults: analysis from the Coronary Artery Risk Development in Young Adults cohort. Ann Rheum Dis. 2013;72:1321-7.

14. Zhang W, Sun K, Yang Y, Zhang H, Hu FB, Hui R. Plasma uric acid and hypertension in a Chinese community: prospective study and metaanalysis. Clin Chem. 2009;55:2026-34.

15. Sundström J, Sullivan L, D'Agostino RB, Levy D, Kannel WB, Vasan RS. Relations of serum uric acid to longitudinal blood pressure tracking and hypertension incidence. Hypertension. 2005;45:28-33.

16. Qi SF, Zhang B, Wang HJ, Yan J, Mi YJ, Liu DW, et al. Prevalence of hypertension subtypes in 2011 and the trends from 1991 to 2011 among Chinese adults. J Epidemiol Community Health. 2015. doi:10.1136/ jech-2015-206492.

17. Yang G, Ma Y, Wang S, Su Y, Rao W, Fu Y, et al. Prevalence and correlates of prehypertension and hypertension among adults in northeastern China: a cross-sectional study. Int J Environ Res Public Health. 2015. doi:10.3390/ ijerph13010082.

18. Masuo K, Kawaguchi H, Mikami H, Ogihara T, Tuck ML. Serum uric acid and plasma norepinephrine concentrations predict subsequent weight gain and blood pressure elevation. Hypertension. 2003:42:474-80.

19. Zhang W, Li N. Prevalence, risk factors, and management of prehypertension. Int J Hypertens. 2011;2011:605359.

20. Jiang L, Mo D, Yang R, Ye Q, Wu J, Yu G, et al. Joint effects of serum uric acid and body mass index on risk of prehypertension in Chinese population. Clin Chim Acta. 2015:446:1-5. 